\title{
Introducing the One-Pot-Amine-Thiol-Ene Reaction to Develop Redox Responsive Nanocarriers for its Potential Use in Anticancer Drug Release
}

\section{Sayan Basak*}

Department of Polymer Science and Technology, University of Calcutta, Kolkata, West Bengal, India

*Corresponding Author: Sayan Basak, Department of Polymer Science and Technology, University of Calcutta, Kolkata, West Bengal, India.
Received: March 20, 2020

Published: April 16, 2020

(C) All rights are reserved by Sayan Basak
The aim to protect the healthy cells remains one of the prime challenges while designing an anticancer drug and its delivery pathway. With the advent of advanced nanocarriers coupled with stimuli-responsive polymer frameworks, the drug-loaded matrix can efficiently deliver the drug with a negligible impact on the healthy cells. The reinforced linkages (ionic and covalent) between the drug molecule and the polymer help in an enhanced encapsulation, thereby minimizing the faulty delivery and increasing the overall efficacy of the process. Furthermore, the integration of stimuli-responsive properties tunes the material to exhibit more precise functionalities, such as reputing of the polymer network at the desired location when excited with a specific stimulus, harmonizes the process to deliver drug molecules very precisely.

Recently, the zwitterions based polymer matrices have emerged to be one of the potent candidates in fabricating functional materials supplementing the efficacy of the nanocarriers. Apart from being superhydrophobic (one of the prime criteria to devise a nanocarrier which can attach a higher amount of drug molecules to deliver), these novel materials have high biocompatibility along with antifouling traits. Furthermore, the charge conversion of zwitterionic polymers provides various complementary advantages. While the negatively charged polymers can improve the total duration of blood circulation (hence increase intracellular uptake into tumor sites), the positively charged polymers act as the 'sticking' signals for detecting cancer breast cells.

This article aims to provide a brief spotlight on the recent works communicated by Lim and his research group with the objectives to develop a zwitter ion-based redox-active polymer system by one-pot-amine-thiol-ene reaction for anticancer drug release applications. The research conducted by Prez., et al. in synthesizing alternating functional polymers via the one-pot-amine-thiol-ene conjugation opened up a variety of avenues to develop multifunctional polymers.

Inspired from the previous works, the current technique, for the first time, employs one-pot thiolactone chemistry to fabricate redox responsive nanogels fortified upon poly (sulfobetaine)s (PSB) (Figure 1). Atom transfer radical polymerization (ATRP) was employed to build the PSB backbones using bromo-2-methyl-propanoate containing furan-maleimide adducts as a micro initiator. Both the proton NMR spectroscopy and the FTIR spectroscopy confirmed the formation of the PSB backbone. Consequently, the 'click chemistry' was integrated by conjugating alkyne-functionalized thiolactones with the PSB backbone. The aminolysis reaction with the cystamine cross-linkers accelerated the process of opening up of the thiolactone rings present in the functionalized PSB. The three-dimensional network was formed when the free thiol groups reacted with the double bonds of the furan maleimide via thiol-ene click reaction when excited under UV irradiation. FTIR spectroscopy confirmed that the reduction of the thioester carbonyl absorbance peak at $1696 \mathrm{~cm}-1$ coupled with an increase in the amide carbonyl peak at $1650 \mathrm{~cm}-1$. Furthermore, the ratio between the C-O stretching bands due to the carboxylate groups at $1696 \mathrm{~cm}-1$ and $1200 \mathrm{~cm}-1$ reduced significantly after the cross-linking took place.

The antifouling properties of the fabricated PSB nanogels were examined via the Bovine serum albumin adsorption test and contrasted with pristine PSB and the conventionally used PEG matrix. The assay revealed that PSB nanogels and the pure PBA exhibited $27.6 \%$ and $29.5 \%$ absorption of Bovine serum albumin (at $286 \mathrm{~nm}$ ), respectively, while PEG recorded $53.4 \%$. The better performance of the PSB nanogels, as compared to PEG, was due to the differences in the polymer hydration, which induced a minimal interaction with hydrophobic protein surface patches.

The in vitro drug release profile with DOX was studied, and it was found that the material could achieve a high drug loading capacity with $24.3 \mathrm{wt} \%$ of the loaded cargo. It was also found that on increasing the cross-linking of the matrix, the drug lading capacity was also enhanced. Increasing the cross-linking implies a growth in the number of the hydrophobic part (cystamine), which leads to a stronger interaction and binding capacity between the DOX and the nanogels. The amalgamation of the positive charges (DOX) and the 
negative charges (PSB gels) develop the ionic binds, which opens rooms for the incorporation of more DOX moieties. As a concluding remark, we can assert that the elevation of the drug loading capacity is cooperated by both the ionic and the hydrophobic interactions.

The degradation of the nanogels was analyzed by evaluating the hydrodynamic size of the nanogels via incorporating glutathione at different concentrations. The reduction induced the S-S bond cleavage of the disulfide cross-linker present in cystamine via the thiol-disulfide exchange reaction. The release rate in presence of glutathione increased with an elevation in the concentration of the glutathione. There are two primary reasons which may describe the above phenomenon. Although, the primary one being the decomposition of the S-S linkages; the transition of the ionic bonding between the negatively charged nanogels and the possibly charged amino acids in the DOX catalyzed by glutathione also helps the release rate rise.

The article provides a fundamental analysis of using zwitterionic based polymer matrix as a nanocarrier for novel drug delivery practices. The high loading efficacy, along with the in vitro release profile of the drug, widens several opportunities for designing highly precise and stimuli-responsive drug nanocarriers [1].

\section{Bibliography}

1. Quoc Thang Phan., et al. "Synthesis of zwitterionic redoxresponsive nanogels by one-pot amine-thiol-ene reaction for anticancer drug release application". Reactive and Functional Polymers 147 (2020): 104463.

\section{Assets from publication with us}

- Prompt Acknowledgement after receiving the article

- Thorough Double blinded peer review

- Rapid Publication

- Issue of Publication Certificate

- High visibility of your Published work

Website: www.actascientific.com/

Submit Article: www.actascientific.com/submission.php

Email us: editor@actascientific.com

Contact us: +919182824667

Citation: Sayan Basak. "Introducing the One-Pot-Amine-Thiol-Ene Reaction to Develop Redox Responsive Nanocarriers for its Potential Use in Anticancer Drug Release”. Acta Scientific Paediatrics 3.4 (2020): 03-04. 